\title{
Impact of Gross Strap Muscle Invasion on Outcome of Differentiated Thyroid Cancer: Systematic Review and Meta-Analysis
}

\author{
Running Title: Impact of gSMI in DTC
}

Li Zhang ${ }^{1}$, MD, Jia Liu ${ }^{2}$, MD,PhD, Peisong Wang ${ }^{2}$, MD,PhD, Shuai Xue ${ }^{2}, \mathrm{MD}^{*}$, Jie $\mathrm{Li}^{3}, \mathrm{MD}, \mathrm{PhD}^{*}, \mathrm{Guang} \mathrm{Chen}^{2}, \mathrm{MD}, \mathrm{PhD}$

1:Department of Nephrology, 2:Department of Thyroid Surgery, 3:Department of Geriatric, The 1st hospital of Jilin University, Changchun, Jilin 130021, People's Republic of China

*Corresponding authors:

Shuai Xue, Department of Thyroid Surgery, The 1st hospital of Jilin University, Changchun, Jilin 130021, People's Republic of China. Tel: 86-18243073755, Email: xueshuai@jlu.edu.cn

Jie Li, Department of Geriatric, The 1st hospital of Jilin University, Changchun, Jilin 130021, People's Republic of China. Tel: 86-13844089236, Email: yabianwanghai@163.com

All the authors declare that they have no competing interests

Key words: strap muscle invasion; prognosis; differentiated thyroid carcinoma; meta-analysis; review; 


\begin{abstract}
:
Background: Gross strap muscle invasion (gSMI) in patients with differentiated thyroid cancer (DTC) was defined as high-risk recurrent group in the 2015 American Thyroid Association guidelines. However, controversy persists because several studies suggested gSMI had little effect on disease outcome. Herein, a systematic review and meta-analysis was conducted to investigate impact of gSMI on outcome of DTC.
\end{abstract}

Methods: A systematic search of electronic databases (PubMed, EMBASE, Cochrane Library, and MEDLINE) for studies published until February 2020 was performed. Case-control studies and randomized controlled trials that studied the impact of gSMI on outcome of DTC were included.

Results: Six studies (all retrospective studies) involving 13639 patients met final inclusion criteria. Compared with no extrathyroidal extension (ETE), patients with gSMI were associated with increased risk of recurrence $(P=0.0004, \mathrm{OR}, 1.46 ; 95 \% \mathrm{CI}$ : 1.18 to 1.80$)$ and lymph node metastasis (LNM) $(P<0.00001$, OR 4.19;95\% CI. 2.53 to 6.96). For mortality ( $P=0.34, \mathrm{OR} 1.47 ; 95 \% \mathrm{CI}: 0.67$ to 3.25$)$, ten-year disease-specific survival ( $P=0.80$, OR $0.91 ; 95 \%$ CI:0.44 to 1.88$)$ and distant metastasis (DM) $(P=0.21$, OR $2.94 ; 95 \%$ CI. 0.54 to 15.93 ), there was no significant difference between gSMI and no ETE group. In contrast with maximal ETE, patients with gSMI were associated with decreased risk of recurrence $(P<0.0001, \mathrm{OR}, 0.58 ; 95 \% \mathrm{CI}: 0.44$ to 0.76 ) , mortality ( $P=0.0003$,OR $0.20 ; 95 \%$ CI:0.08 to 0.48$)$, LNM ( $P=0.0003$,OR $0.64 ; 95 \%$ CI. 0.50 to 0.81$)$ and DM ( $P=0.0009$,OR $0.28 ; 95 \%$ CI. 0.13 to 0.59$)$.

Conclusions: DTC patients with gSMI had a higher risk of recurrence and LNM than those without ETE. However, in contrast with maximal ETE, a much better prognosis was observed in DTC patients with only gSMI. The findings of our meta-analysis provide supportive evidence for the validity of the $T$ category changes in the 8th edition American Joint Committee on Cancer system. The actual impact of gSMI should be re-evaluated and revised in the recurrent risk stratification system in the future. 


\section{Introduction}

Extrathyroidal extension (ETE), which is defined as tumor spread outside of the thyroid gland and into the surrounding tissues, occurs in up to $30 \%$ of patients with differentiated thyroid cancer (DTC) [1, 2]. Minimal ETE (mETE), detectable only on histological examination, was not regarded as a negative predictor for either survival or disease recurrence[3-5]. Accordingly, mETE was removed from the T3 definition in the 8th edition of the American Joint Committee on Cancer (AJCC) classification, as it would not affect either T category or overall stage[6]. In contrast, gross ETE is believed to be an important risk factor for recurrence and mortality[7, 8]. Thus, DTC patients with gross ETE are classified as T3b or T4 in the AJCC system[9]. Moreover, the 2015 American Thyroid Association (ATA) guidelines grouped tumors with gross ETE in the high risk of recurrence category, with anearly $20 \%$ risk of structural recurrence[10]. Therefore, gross ETE was an absolute indication for total thyroidectomy and the administration of postoperative radioactive iodine.

In addition to the degree of gross ETE, the site of gross tumor invasion also plays important roles in disease-specific survival (DSS) and disease-free survival (DFS). Recently, several studies reported that gross strap muscle invasion (gSMI) had little effect on DSS and DFS, which was different from the findings of previous studies[8, 11]. Increasing evidence suggests that DTC patients with only gSMI have the same DFS as those with microscopic ETE[11]. In our previous study, we also found that only 4 of $30(13.3 \%)$ Braf-mutated thyroid papillary microcarcinoma patients with gSMI were diagnosed with recurrence[12]. Accordingly, Shaha AR suggested that a detailed distinction of gross ETE should be performed[13]. Patients with anterior ETE involving the strap muscle had a relatively good prognosis compared with those with posterior gross ETE to the recurrent laryngeal nerve, trachea or esophagus[8]. A possible reason is that gSMI can be easily resected with negative margins[13].

In light of the conflicting data on the recurrence risk and mortality conferred by gSMI, we performed a systematic review and meta-analysis to assess the impact of gSMI on the outcomes of DTC patients. 


\section{Materials and Methods}

This meta-analysis was conducted in accordance with the Cochrane Handbook for Systematic Reviews of Interventions guidelines[14]. There was no funding received for this study.

\subsection{Search strategy}

We searched the EMBASE, PubMed, MEDLINE, and Cochrane databases from inception to February 26, 2020, without any language limitations. The following terms were used in the search: "thyrohyoid muscle", "sternothyroid muscle", "sternohyoid muscle", "strap muscle", "gross extrathyroidal extension", "invasion", "extension", "thyroid cancer*", "thyroid carcinoma*”, and "thyroid neoplasm*".

\subsection{Inclusion and exclusion criteria}

The studies returned from the search were checked according to the following inclusion criteria: (1) patients were more than 18 years old; (2) pathologically proven DTC patients who underwent surgery; (3) complete clinical data and follow-up information; and (4) DTC patients with SMI.

The exclusion criteria were as follows: (1) patients with less than 12 months of follow-up; (2) those with incomplete medical records; (3) those with medullary thyroid carcinoma and undifferentiated carcinoma; and (4) publication styles were letters to the editor, abstracts and meeting posters.

\subsection{Data Extraction and Risk of Bias Assessment}

L Zhang and J Liu assessed the search results according to the relevance in providing information for the review. Two reviewers (L Zhang and S Xue) independently assessed the titles and abstracts of the remaining records for relevance according to the protocol criteria. Then, they browsed the full text of the studies in detail. Any disagreements were resolved by consulting a third reviewer (J Li). L Zhang assessed the risk of bias of each included study using the relevant, validated tool for each study 
design. J Liu performed the risk of bias assessment. The risk of bias of the included trials was assessed using the Newcastle-Ottawa Scale[15].

\subsection{Statistical analysis}

Review Manager (RevMan) 5.3 software was used for the analysis. We calculated odds ratios (ORs) with 95\% confidence intervals (CIs) for dichotomous data. We assessed the heterogeneity across studies using the $\mathrm{Q}$ test and the $\mathrm{I}^{2}$ statistic. $\mathrm{P}<0.1$ and $\mathrm{I}^{2}>50 \%$ indicated statistical significance[16]. If there was obvious heterogeneity, we used a random-effects model; otherwise, we used a fixed-effects model. We conducted sensitivity analysis by excluding each single study at a time to test its influence on the pooled effects. The source of heterogeneity was also explored by subgroup analyses of operation type and histopathological subtype based on available information. When the p-value was less than 0.05 , it was considered statistically significant.

\section{Results}

\subsection{Literature Search}

We initially identified a total of 219 studies. Fifty-two duplicate studies and another 118 studies were excluded after reviewing the titles and abstracts. After scrutiny of the full texts of the remaining 49 articles, six studies were finally included in this meta-analysis, all of which were retrospective studies[8, 11, 17-20]. Fig 1 shows the study selection process. 


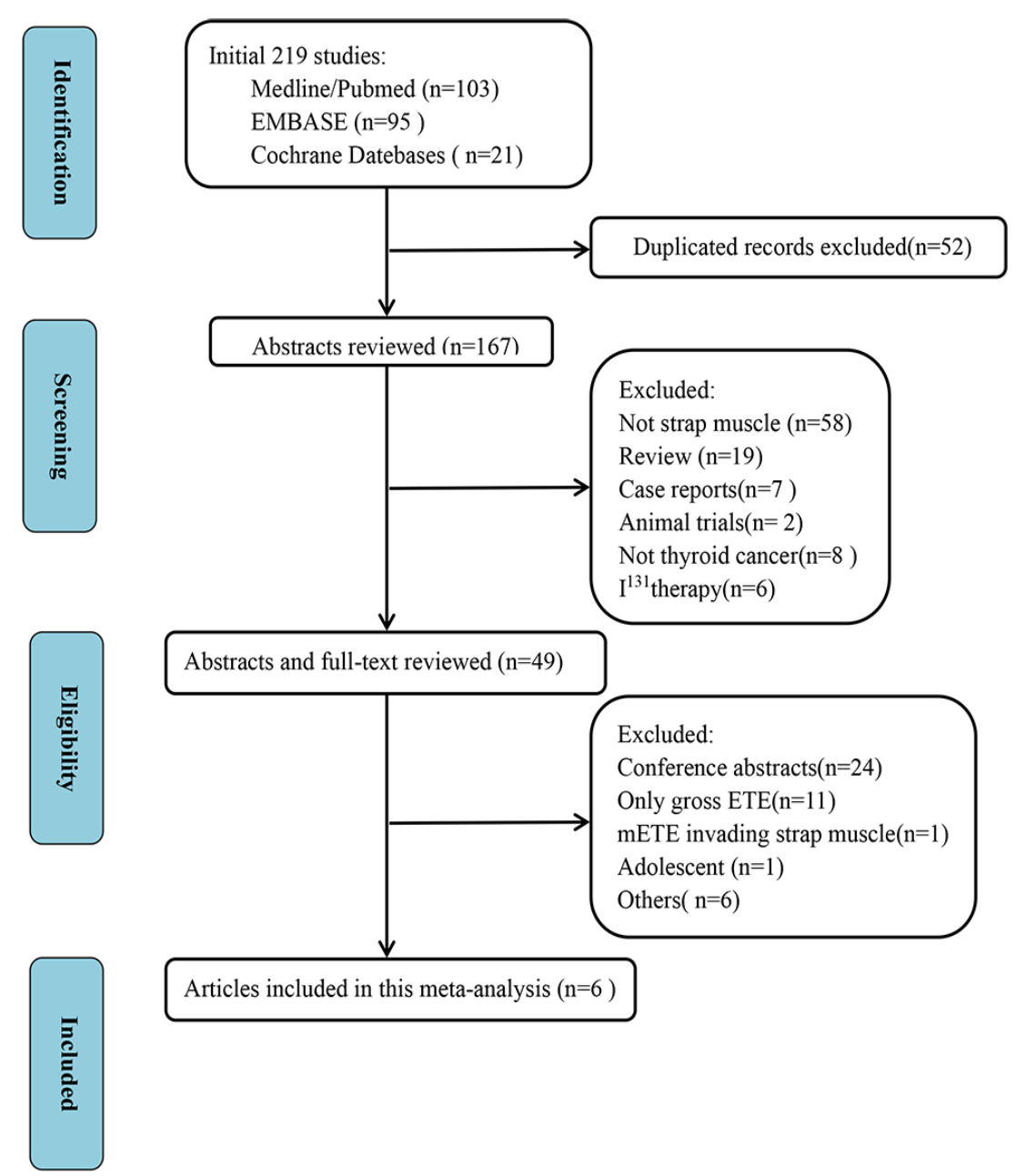

Fig 1: Selection process of studies in meta-analysis

\subsection{Study Characteristics and Quality}

In this meta-analysis, a total of 13639 patients were included, and the characteristics of the included studies are presented in Table 1. The maximal ETE means extension of the primary tumor to the trachea, esophagus, recurrent laryngeal nerve, larynx, subcutaneous soft tissue, skin, internal jugular vein, or carotid artery. No ETE means no extrathyroidal extension. The quality assessment of the included studies by the Newcastle-Ottawa Scale is presented in Table 2. All studies used hospital controls, who were accessed by the same method as gross ETE into the strap muscle (gETE st+ group). Multivariate analysis was conducted by all the studies. The scores of all the studies were over 5; thus, the quality of the selected studies was generally high. 


\section{Table1:Baseline Characteristics of included studies}

\begin{tabular}{|c|c|c|c|c|c|c|c|c|c|c|}
\hline Study & $\begin{array}{c}\text { Patients } \\
\text { No. }\end{array}$ & Groups & $\begin{array}{c}\text { Patient } \\
\text { No. }\end{array}$ & $\begin{array}{c}\text { Female, } \\
\text { n, \% }\end{array}$ & $\begin{array}{c}\text { Age (y) } \\
\text { Mean (SD) } \\
\text { /Median (rang) }\end{array}$ & $\begin{array}{l}\text { Tumor Size }(\mathbf{m m}) \\
\text { Mean }(\mathrm{SD}) \\
\text { /Median (rang) }\end{array}$ & $\operatorname{RAI}(\mathbf{n}, \%)$ & $\begin{array}{c}\text { Multifocality } \\
(\mathbf{n}, \%)\end{array}$ & N Stage & $\begin{array}{l}\text { Follow-Up } \\
\text { Mean (SD) } \\
\text { /Median (rang) }\end{array}$ \\
\hline \multirow{4}{*}{ Amit,2018 } & \multirow{3}{*}{2084} & no ETE (group 1) & 1291 & $966(75)$ & $47(18-87)$ & \multirow{3}{*}{ N/A } & $555(44)$ & $479(37)$ & \multirow{3}{*}{$\begin{array}{c}\text { Nx N0 } \\
\text { N1a N1b }\end{array}$} & $66(12-192)$ \\
\hline & & ETE into perithyroidal soft tissue (group 2) & 732 & $527(72)$ & $47(18-97)$ & & $527(74)$ & $397(54)$ & & $51(12-189)$ \\
\hline & & gETE into strap muscle (group 3) & 61 & $52(85)$ & $48(19-83)$ & & $48(78)$ & $29(48)$ & & $64(17-155)$ \\
\hline & \multirow{4}{*}{4045} & no ETE & 2300 & $1679(73.0)$ & $42.6(10.5)$ & $11.17(8.11)$ & $928(40.3)$ & $625(27.1)$ & & $30(12-63)$ \\
\hline \multirow{2}{*}{$\mathrm{Li}, 2018$} & & ETE into perithyroidal tissue & 1004 & $726(72.3)$ & $43.2(12.1)$ & 13.64(9.61) & $634(63.2)$ & 274(27.3) & \multirow{3}{*}{ N/A } & $34(12-63)$ \\
\hline & & T3b (gETE into the strap muscles) & 371 & $286(77.1)$ & $44.1(10.4)$ & $18.42(10.61)$ & $325(87.6)$ & 124(33.4) & & $32(13-58)$ \\
\hline \multirow{6}{*}{ Park,2018 } & & ETE beyond the strap muscles & 370 & $262(70.8)$ & $46.3(11.1)$ & $21.15(9.88)$ & $361(97.6)$ & $134(36.2)$ & & $30(12-59)$ \\
\hline & \multirow{5}{*}{3174} & no ETE & 1362 & $1170(85.9)$ & $45.5(11.6)$ & $12.5(11.0)$ & $1024(75.2)$ & \multirow{4}{*}{ N/A } & \multirow{4}{*}{ N0 N1 } & \multirow{4}{*}{$148.8(133.2-174)$} \\
\hline & & microscopic ETE & 1377 & $1199(87.1)$ & $46.2(12.2)$ & 15.3(11.9) & $1299(94.3)$ & & & \\
\hline & & gETE invading only strap muscles & 261 & $227(87.0)$ & $49.0(13.1)$ & $19.1(13.5)$ & $246(94.3)$ & & & \\
\hline & & gETE invading perithyroidal structures & 174 & $150(86.2)$ & $52.4(15.0)$ & $24.2(18.7)$ & $153(87.9)$ & & & \\
\hline & & $\mathrm{T} 1$ & 1997 & \multirow{5}{*}{ 2712(87.4) } & \multirow{5}{*}{$45.9(37.8-54.2)$} & \multirow{5}{*}{$13(8-22)$} & \multirow{5}{*}{$2363(76.1)$} & \multirow{6}{*}{ N/A } & \multirow{6}{*}{ N0 N1 } & \multirow{5}{*}{$120(97.2-144)$} \\
\hline \multirow{4}{*}{ Song, 2018} & \multirow{4}{*}{3104} & $\mathrm{~T} 2$ & 496 & & & & & & & \\
\hline & & $\mathrm{Y} 3 \mathrm{a}$ & 96 & & & & & & & \\
\hline & & $\mathrm{T} 3 \mathrm{~b}(\leq 4 \mathrm{~cm}$ and $\mathrm{gETE}$ to strap muscle $)$ & 376 & & & & & & & \\
\hline & & $\mathrm{T} 3 \mathrm{~b}(>4 \mathrm{~cm}$ and $\mathrm{gETE}$ to strap muscle $)$ & 38 & & & & & & & \\
\hline \multirow{4}{*}{ Song,2019 } & \multirow{3}{*}{636} & $\mathrm{~T} 4 \mathrm{a}$ & 101 & & & & & & & \\
\hline & & without gETE & 586 & $457(78.0)$ & $45.0(11.4)$ & $12(10-15)$ & \multirow{2}{*}{ N/A } & $53(9.0)$ & \multirow{2}{*}{ N0 N1a } & $91.2(61.2-130.8)$ \\
\hline & & those with gETE to strap muscle & 50 & $45(90.0)$ & $49.9(8.9)$ & $12(11-15)$ & & $7(14.0)$ & & $84(60-102)$ \\
\hline & 596 & low-risk PTC without ETE (low w/o ETE) & 251 & $231(92)$ & $50.8(12)$ & $14.9(16.4)$ & $102(40.6)$ & $107(43)$ & N0 N1a & $48(12-322)$ \\
\hline
\end{tabular}




\begin{tabular}{|c|c|c|c|c|c|c|c|c|}
\hline \multirow[t]{3}{*}{ Danilovic, 2019} & $\begin{array}{l}\text { intermediate-risk PTC without ETE } \\
\text { (intermediate w/o ETE) }\end{array}$ & 89 & $76(85.4)$ & $44.9(15.8)$ & 24.1(18.9) & $81(91)$ & $57(64.8)$ & $43.2(12-217)$ \\
\hline & $\operatorname{minimal}(\mathrm{mETE})$ & 191 & $166(86.9)$ & $50.0(13.7)$ & $17.3(13.4)$ & $186(97.4)$ & $121(63.4)$ & $39.6(12-142)$ \\
\hline & gETE into the strap muscles (gETE) & 65 & $54(83.1)$ & $51.6(14.5)$ & $28.0(16.0)$ & 64 (98.5) & $35(54.7)$ & $43.2(12-260)$ \\
\hline
\end{tabular}

Abbreviations:N/A:Not applicable; SD: Standard Deviation;ETE extrathyroidal extension;gETE:gross extrathyroidal extension;RAI;radioactive iodine;LN lymph node;RLN:recurrent laryngeal nerve. 
Table 2. Quality assessment of the included studies by the Newcastle-Ottawa Scale

\begin{tabular}{|c|c|c|c|c|c|c|}
\hline Items & $\begin{array}{l}\text { Amit } \\
2018\end{array}$ & $\begin{array}{c}\mathrm{Li} \\
2018 \\
\end{array}$ & $\begin{array}{l}\text { Park } \\
2018\end{array}$ & $\begin{array}{l}\text { Song } \\
2018 \\
\end{array}$ & $\begin{array}{l}\text { Song } \\
2019 \\
\end{array}$ & $\begin{array}{c}\text { Danilovic } \\
2019 \\
\end{array}$ \\
\hline \multicolumn{7}{|l|}{ Selection } \\
\hline Is the case definition adequate? & 1 & 1 & 1 & 1 & 1 & 1 \\
\hline Representativeness of the cases & 1 & 1 & 1 & 1 & 1 & 1 \\
\hline Selection of Controls & 0 & 0 & 0 & 0 & 0 & 0 \\
\hline Definition of Controls & 1 & 1 & 1 & 1 & 1 & 1 \\
\hline $\begin{array}{l}\text { Comparability of cases and controls } \\
\text { on the basis of the design or analysis }\end{array}$ & 2 & 2 & 2 & 2 & 2 & 2 \\
\hline \multicolumn{7}{|l|}{ Outcome } \\
\hline Ascertainment of exposure & 1 & 1 & 1 & 1 & 1 & 1 \\
\hline $\begin{array}{l}\text { Same method of ascertainment for } \\
\text { cases and controls }\end{array}$ & 1 & 1 & 1 & 1 & 1 & 1 \\
\hline Non-Response rate & 0 & 0 & 1 & 0 & 0 & 0 \\
\hline Total score & 7 & 7 & 8 & 7 & 7 & 7 \\
\hline
\end{tabular}

\subsection{Outcomes}

\subsubsection{Locoregional recurrence (LRR)}

Five studies evaluated the impact of gSMI on recurrence in 6687 patients with DTC (no ETE in 5879 subjects, gETE st+ in 808 subjects). gSMI in patients was associated with an increased risk of recurrence $(P=0.0004 ; \mathrm{OR}, 1.46 ; 95 \% \mathrm{CI}: 1.18$ to 1.80$)$ without heterogeneity $\left(\mathrm{I}^{2}=0 \%\right)$ (Fig. $\left.2 \mathrm{~A}\right)$. Two studies compared the impact of gSMI on recurrence with maximal ETE. Compared with maximal ETE, gSMI was associated with a decreased risk of recurrence $(P<0.0001$; OR, $0.58 ; 95 \%$ CI: 0.44 to 0.76) without heterogeneity $\left(\mathrm{I}^{2}=0 \%\right)$ (Fig. $\left.2 \mathrm{~B}\right)$. At the same time, two studies compared the impact of gSMI on recurrence with perithyroidal soft tissue invasion. The locoregional recurrence between patients with gSMI and those with perithyroidal soft tissue invasion was not significantly different $(P=0.07$; OR, $1.28 ; 95 \%$ CI: 0.98 to 1.68) without heterogeneity $\left(\mathrm{I}^{2}=0 \%\right)$ (Fig. 2C). 


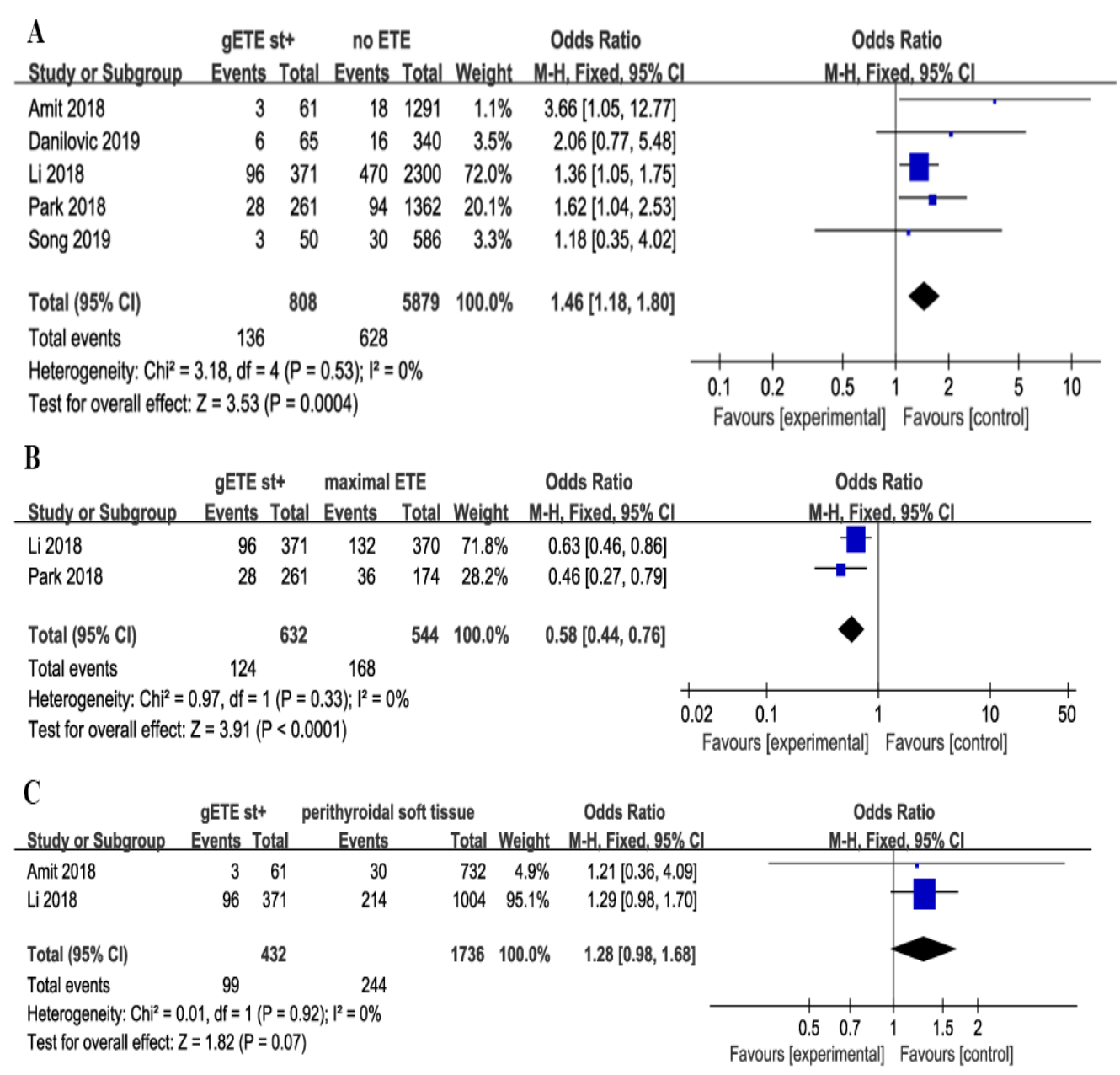

Fig 2: Forest plot for LRR comparison between gETE st+ with (A) no ETE (B) maximal ETE (C) perithyroidal soft tissue

\subsubsection{Overall mortality}

Three studies compared the impact of gSMI on cancer-related mortality with no ETE group among 4699 patients with DTC (no ETE in 4002 subjects, gETE st+ in 697 subjects). Two studies compared the impact of gSMI on cancer-related mortality with the maximal ETE group. The mortality of patients with gSMI was not increased compared with that of no ETE patients ( $P=0.34$; OR, 1.47; 95\% CI: 0.67 to 3.25) (Fig. 3A). Compared with maximal ETE, gSMI was associated with decreased mortality $(P=0.0003$; OR, 0.20 ; 95\% CI: 0.08 to 0.48$)$ (Fig. 3B). 


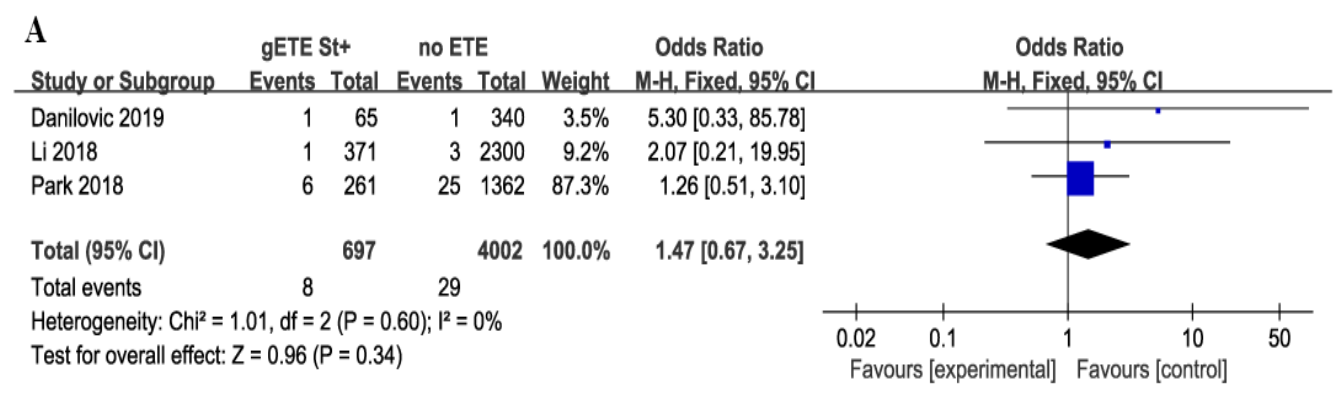

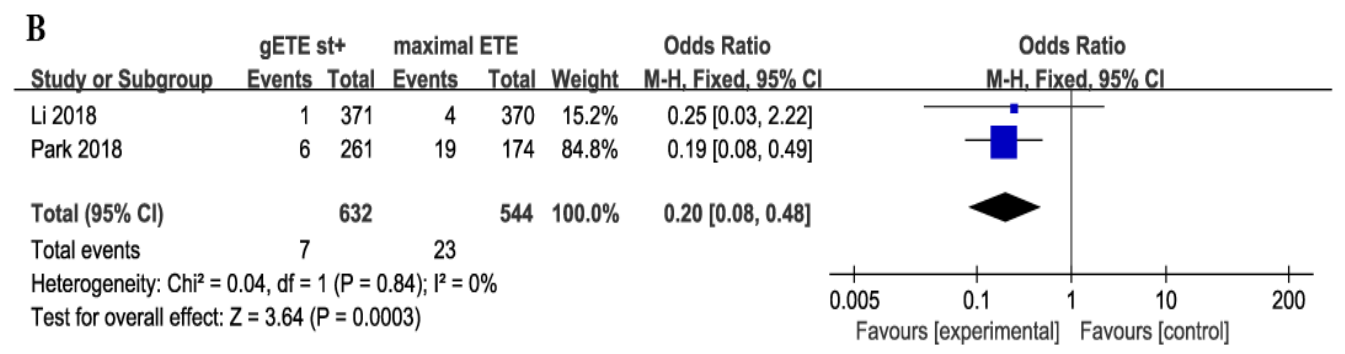

Fig 3: Forest plot for overall mortality comparison between gETE st+ with (A) no ETE (B) maximal ETE

\subsubsection{Ten-year Disease-specific Survival}

Three studies analyzed the impact of gSMI and no ETE on ten-year DSS among 3981 patients with DTC. There was no significant difference between the no ETE and gSMI groups $(P=0.80 ;$ OR, $0.91 ; 95 \% \mathrm{CI}: 0.44$ to 1.88$)$ with no heterogeneity $\left(\mathrm{I}^{2}=0 \%\right)$ (Fig. 4).

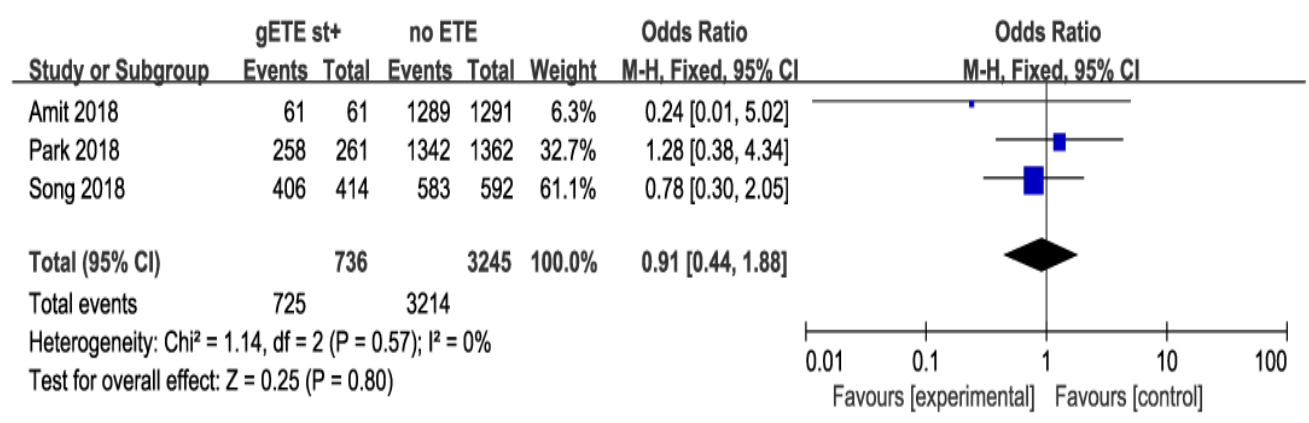

Fig 4: Forest plot for ten-year DSS comparison between gETE st+ with no ETE

\subsubsection{Lymph Node Metastases}

Four studies compared the impact of gSMI and no ETE on baseline lymph node metastases (LNM) among 6051 patients. gSMI was associated with an elevated LNM ratio $(P<0.00001 ; \mathrm{OR}, 4.19 ; 95 \% \mathrm{CI} .2 .53$ to 6.96$)$ with significant heterogeneity 
$\left(\mathrm{I}^{2}=86 \%\right.$ ) (Fig. 5A). Moreover, only two studies investigated the impact of gSMI and maximal ETE on LNM among 1176 patients. Compared with maximal ETE, gSMI in patients was associated with decreased LNM ( $P=0.0003$; OR, $0.64 ; 95 \%$ CI. 0.50 to 0.81) (Fig. 5B).

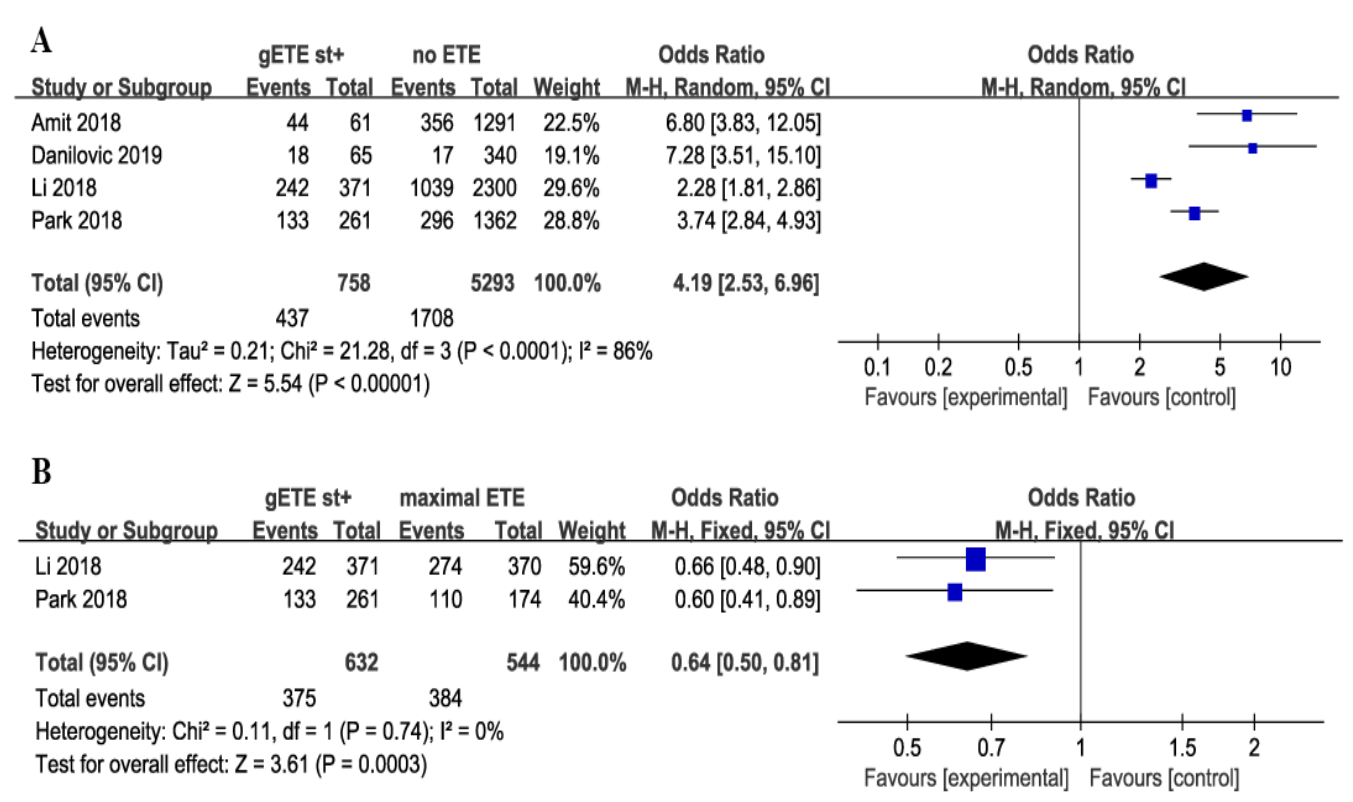

Fig 5: Forest plot for LNM between gETE st+ with (A) no ETE (B) maximal ETE

\subsubsection{Distant Metastases}

Four studies assessed the impact of gSMI and no ETE on distant metastases (DM). There was no significant difference in the DM ratio between the gSMI and no ETE groups $(P=0.21)$, with significant heterogeneity $\left(\mathrm{I}^{2}=87 \%\right)$ (Fig. 6A). Moreover, only two studies investigated the impact of gSMI and maximal ETE on DM. gSMI in patients was associated with decreased DM ( $P=0.0009$; OR, $0.28 ; 95 \%$ CI. 0.13 to 0.59) with no heterogeneity $\left(\mathrm{I}^{2}=19 \%\right)$ (Fig. 6B). 

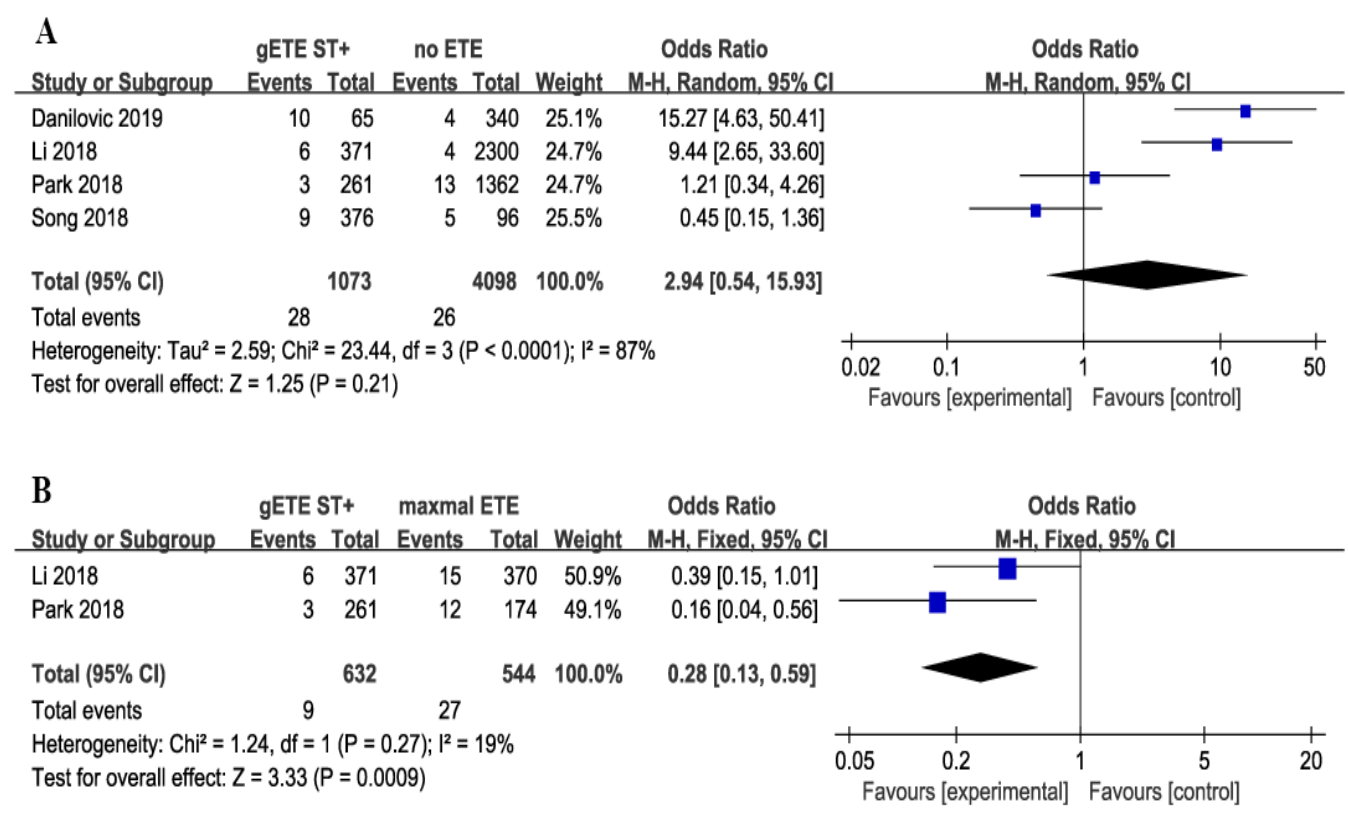

Fig 6: Forest plot for DM comparison between gETE st+ with (A) no ETE (B) maximal ETE

\subsection{Sensitivity analysis and subgroup analysis}

For the comparisons with significant heterogeneity, we conducted sensitivity analysis.

The leave-one-out meta-analysis revealed that LNM (compared with no ETE) and DM (compared with no ETE) did not identify a single study that may have caused the substantial heterogeneity. (Data not shown)

Furthermore, the degree of LNM is highly dependent on the type of lymph node dissection (LND). Prophylactic LND will identify many microscopic LNM, while therapeutic LND is only performed for patients with clinical metastatic lymph nodes. All and some patients underwent prophylactic LND in the Li and Park studies, respectively. Therapeutic LND was performed in the studies by Danilovic and Amit. In the therapeutic LND subgroup, patients with gSMI had increased LNM compared with patients without ETE. $\left(P<0.00001\right.$; OR, 6.94; 95\% CI. 4.40 to $\left.10.95 ; \mathrm{I}^{2}=0 \%\right)$ (Fig. 7). 


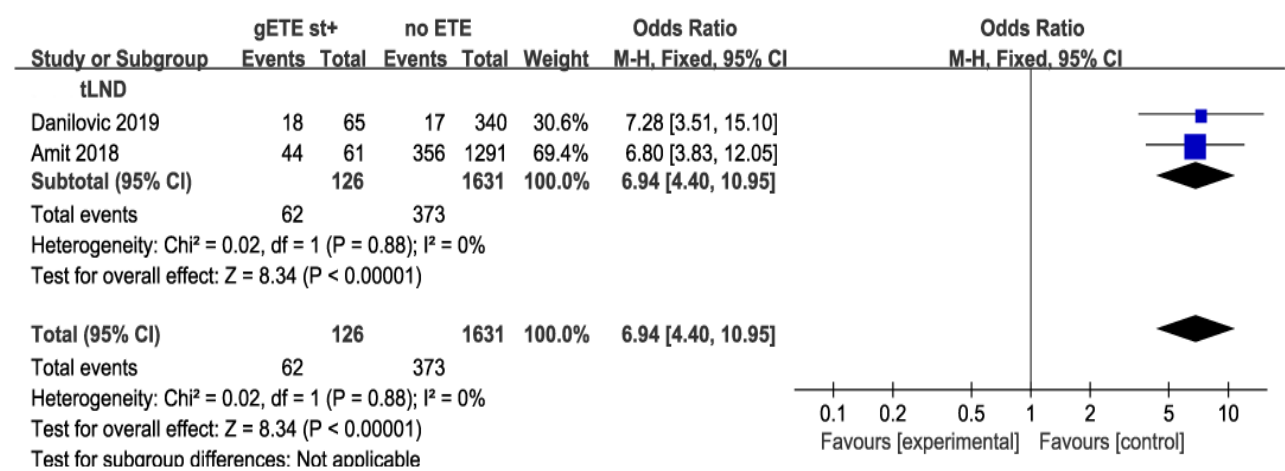

Fig 7: Subgroup analysis for LNM comparison between gETE st+ with no ETE

Patients with follicular thyroid carcinoma are more likely to present with DM than those with papillary thyroid carcinoma (PTC). We believe the significant heterogeneity of the DM analysis is mainly attributed to histopathological types. In the DTC subgroup, there was still no significant difference in DM between the gSMI and no ETE groups $(P=0.45)$ without heterogeneity $\left(\mathrm{I}^{2}=25 \%\right)$ (Fig. 8A). However, in the PTC subgroup, we found that gSMI increased DM significantly compared with no ETE $\left(P<0.0001 ;\right.$ OR, 12.35; 95\% CI. 5.20 to $\left.29.29 ; \mathrm{I}^{2}=0 \%\right)$ (Fig. $\left.8 \mathrm{~B}\right)$.

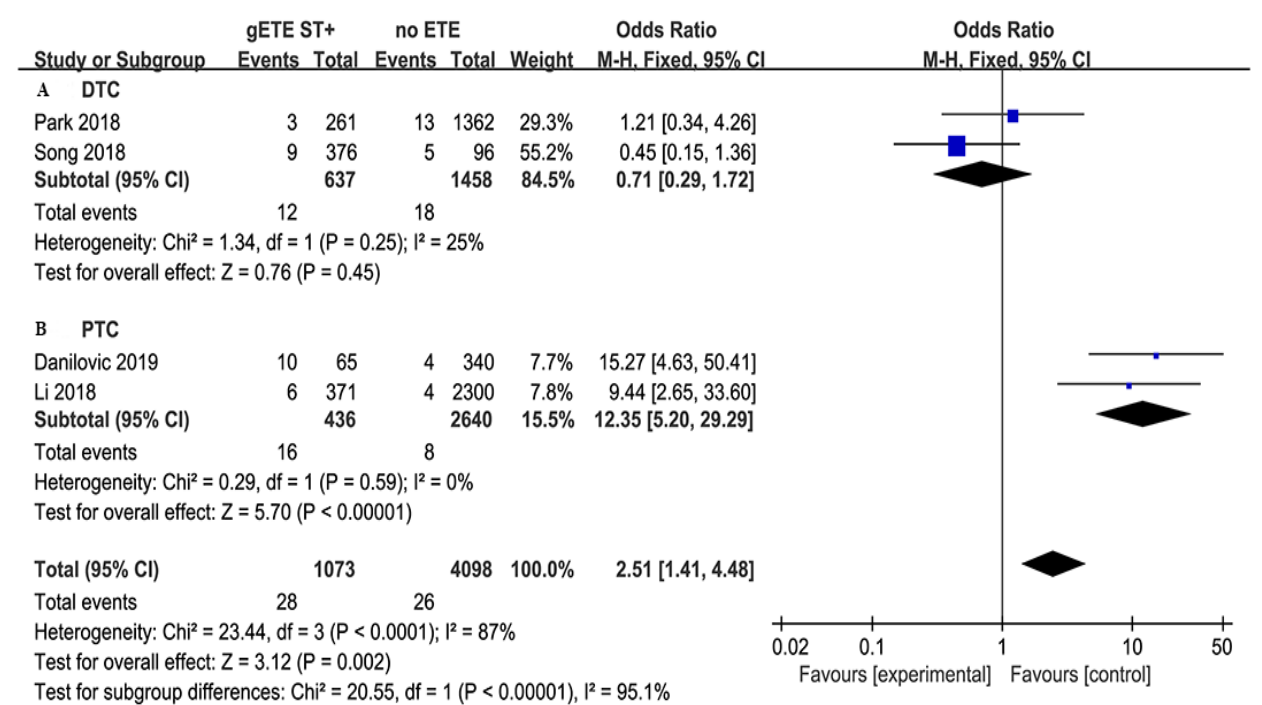

Fig 8: Subgroup analysis for DM comparison between gETE st+ with no ETE

\section{Discussion}

Increasing evidence has shown that the site of gross tumor invasion also plays important roles in the recurrence and mortality of DTC patients[21, 22]. Some 
researchers believed that gSMI had a relatively good prognosis compared with gross ETE to the recurrent laryngeal nerve, trachea or esophagus, which was different from the findings of previous studies[11, 20, 23]. It is still controversial whether DTC with only gSMI should be downgraded to a lower tumor stage and recurrent risk category. To the best of our knowledge, this is the first meta-analysis to assess the impact of gSMI on outcomes in DTC patients. Compared with patients with no ETE, patients with gSMI had an increased risk of recurrence and LNM. For mortality, ten-year DSS and DM, there were no significant differences between the gSMI and no ETE groups. In contrast with those with maximal ETE, patients with gSMI had a decreased risk of recurrence, mortality, LNM and DM.

According to ATA guidelines, tumors with gross ETE are categorized into the high-risk group because of the more than $20 \%$ structural recurrence rate[10]. In our study, the LRR rate of the gSMI group ranged from $5 \%$ to $25.9 \%$. These relatively lower LRR rates were mainly attributed to the exclusion of some high-risk recurrent patients in these studies[11, 19, 20]. In the Danilovic and Li studies, which included all kinds of DTC cases, the LRR rates of gSMI were $24.6 \%$ and $25.9 \%[17,18]$. These data were consistent with the ATA guidelines. Based on the site of tumor invasion, gross ETE can be further divided into 3 subgroups: invasion only to perithyroidal soft tissue, invasion only to strap muscle and invasion beyond the strap muscles (recurrent laryngeal nerve, trachea, esophagus, skin or subcutaneous tissues). We found that DTC patients with gETE beyond the strap muscle suffered a much higher LRR than the other two groups. A possible reason is that gSMI can be easily resected with negative margins[13]. In the future, it may be reasonable that gETE beyond the strap muscle is categorized into an extremely high-risk group in the new recurrence risk stratification system, although further high-quality evidence is needed.

The eighth edition of the AJCC/TNM cancer staging system for DTC was published in 2016[6]. It made a substantial change with regard to the T3 category definition. Because mETE, which is identified only on histological examination, carried much less prognostic importance, the new AJCC/TNM system removed mETE in determining the T category[24]. Moreover, T3b was defined as a tumor of 
any size with gSMI. The 8th edition made clear distinctions of disease with no ETE (T1, T2, T3a), gETE only to the strap muscle (T3b) and gETE beyond the strap muscle (T4)[25]. In our study, we also found that patients with gSMI presented with a higher recurrence rate than patients with no ETE. At the same time, the presence of gETE beyond the strap muscle was associated with a far worse prognosis than that of gSMI. These findings of our meta-analysis provide supportive evidence for the validity of the T category changes in the 8th edition AJCC/TNM system.

Usually, the T stage of tumors is associated with LNM and DM. The invasiveness of tumors represents its severity and differentiation[26]. Patients with aggressive tumors are always accompanied by more LNM and early DM[27]. Compared with patients with no ETE, patients with gSMI present with more LNM. Maximal ETE was considered an independent risk factor for LNM and DM in contrast with gSMI. This finding in our study suggests that the degree of ETE carries much more prognostic significance for DTC[7].

High heterogeneity with an $\mathrm{I}^{2}>50 \%$ was found in the analysis of LNM (compared with no ETE) and DM (compared with no ETE). Additionally, after the removal of each study from the analysis, similar results were confirmed, and the heterogeneity was not changed significantly. Furthermore, subgroup analysis was performed to explore the source of heterogeneity. In the therapeutic LND subgroup, gSMI increased LNM in comparison with ETE. This finding suggested that clinical LNM was more frequent in patients with gSMI. In the PTC subgroup, we found that gSMI increased DM significantly compared with no ETE. Histopathological types may be correlated with the high heterogeneity in the analysis of DM.

\section{Strengths and weaknesses}

By performing a meta-analysis with populations from different studies, this is the first study to assess the impact of gSMI on outcomes in DTC patients in a larger study sample and to adjust the results for the presence of some confounding factors. High heterogeneity was found in the analysis of LNM (compared with no ETE) and DM 
(compared with no ETE) and was compensated by subgroup analysis. The results of LNM (compared with no ETE) and DM (compared with no ETE) should be interpreted with caution because of the limited number of enrolled articles, and further study is needed to confirm the corresponding results.

This meta-analysis has some potential limitations. First, the treatment strategies for DTC patients were different among the enrolled studies. These treatment disparities, such as thyroidectomy, lymph node dissection, radioiodine ablation or follow-up, might contribute to different patient outcomes. Second, the limited number of studies hindered the implementation of meta-regression analysis and publication bias assessment. The results of the subgroup analysis should be interpreted with caution because of the small number of studies, although heterogeneity was eliminated by subgroup analysis. Third, the retrospective and non-randomized nature of all studies included in the analysis might be considered a source of bias. This provided associative, not causal, evidence and mandates caution when interpreting these results. In future studies, randomized controlled trials with a higher methodological quality are needed to improve the quality of evidence.

\section{Conclusion}

Patients with gSMI had a higher risk of recurrence and LNM than those without ETE. However, in contrast with maximal ETE, a much better prognosis was observed in DTC patients with only gSMI. The findings of our meta-analysis provide supportive evidence for the validity of the T category changes in the 8th edition AJCC system. The actual impact of gSMI should be re-evaluated and revised in the recurrent risk stratification system in the future.

\section{Author contributions}

All authors listed have made a substantial, direct and intellectual contribution to the work, and approved it for publication. 


\section{References}

1. Youngwirth LM, Adam MA, Scheri RP, Roman SA, Sosa JA. Extrathyroidal Extension Is Associated with Compromised Survival in Patients with Thyroid Cancer. Thyroid. 2017;27(5):626-631.

2. Jin BJ, Kim MK, Ji YB, Song CM, Park JH, Tae K. Characteristics and significance of minimal and maximal extrathyroidal extension in papillary thyroid carcinoma. Oral Oncol. 2015;51(8):759-63.

3. Diker-Cohen T, Hirsch D, Shimon I, Bachar G, Akirov A, Duskin-Bitan H, Robenshtok E. Impact of Minimal Extra-Thyroid Extension in Differentiated Thyroid Cancer: Systematic Review and Meta-analysis. J Clin Endocrinol Metab. 2018. ;103(6):2100-06.

4. Tam S, Amit M, Boonsripitayanon M, Busaidy NL, Cabanillas ME, Waguespack SG, Gross ND, Grubbs EG, Williams MD, Lai SY, et al. Effect of Tumor Size and Minimal Extrathyroidal Extension in Patients with Differentiated Thyroid Cancer. Thyroid. 2018;28(8):982-90.

5. Al-Qurayshi Z, Shama MA, Randolph GW, Kandil E. Minimal extrathyroidal extension does not affect survival of well-differentiated thyroid cancer. Endocr-Relat Cancer. 2017;24(5):221-6.

6. Perrier ND, Brierley JD, Tuttle RM. Differentiated and anaplastic thyroid carcinoma: Major changes in the American Joint Committee on Cancer eighth edition cancer staging manual. CA Cancer J Clin. 2018;68(1):55-63.

7. Ibrahimpasic T, Ghossein R, Carlson DL, Chernichenko N, Nixon I, Palmer FL, Lee NY, Shaha AR, Patel SG, Tuttle RM, et al. Poorly differentiated thyroid carcinoma presenting with gross extrathyroidal extension: 1986-2009 Memorial Sloan-Kettering Cancer Center experience. Thyroid. 2013;23(8):997-1002.

8. Song E, Lee YM, Oh HS, Jeon MJ, Song DE, Kim TY, Kim WB, Shong YK, Sung TY, Kim WG. A Relook at the T Stage of Differentiated Thyroid Carcinoma with a Focus on Gross Extrathyroidal Extension. Thyroid. 2019;29(2):202-8.

9. Tam S, Boonsripitayanon M, Amit M, Fellman BM, Li YS, Busaidy NL, Cabanillas ME, Dadu R, Sherman S, Waguespack SG, et al. Survival in Differentiated Thyroid Cancer: Comparing the AJCC Cancer Staging Seventh and Eighth Editions. Thyroid. 2018;28(10):1301-10.

10. Haugen BR, Alexander EK, Bible KC, Doherty GM, Mandel SJ, Nikiforov YE, Pacini F, Randolph GW, Sawka AM, Schlumberger M, et al. 2015 American Thyroid Association Management Guidelines for Adult Patients with Thyroid Nodules and Differentiated Thyroid Cancer: The American Thyroid Association Guidelines Task Force on Thyroid Nodules and Differentiated Thyroid Cancer. Thyroid. 2016;26(1):1-133.

11. Amit M, Boonsripitayanon M, Goepfert RP, Tam S, Busaidy NL, Cabanillas ME, Dadu R, Varghese J, Waguespack SG, Gross ND, et al. Extrathyroidal Extension: Does Strap Muscle Invasion Alone Influence Recurrence and Survival in Patients with Differentiated Thyroid Cancer? Ann Surg Oncol. 2018;25(11):3380-8.

12. Xue S, Zhang L, Wang PS, Liu J, Yin Y, Jin MS, Guo L, Zhou YH, Chen G. 
Predictive Factors of Recurrence for Multifocal Papillary Thyroid Microcarcinoma With Braf(v600e) Mutation: A Single Center Study of 1,207 Chinese Patients. Front Endocrinol. 2019;10:9.

13. Shaha AR. Extrathyroidal extension-what does it mean. Oral Oncol. 2017;68:50-2.

14. Higgins JPT TJ, Chandler J, Cumpston M, Li T, Page MJ, Welch VA (editors). Cochrane Handbook for Systematic Reviews of Interventions version 6.0 (updated July 2019). Cochrane, 2019. Available from www.training.cochrane.org/handbook.

15. Wells G SB, O'Connell D, Peterson J. . The Newcastle-Ottawa Scale (NOS) for assessing the quality of nonrandomised studies in meta-analyses 2000.

http://www.ohri.ca/programs/clinical_epidemiology/oxford.asp.

16. Higgins JP, Thompson SG, Deeks JJ, Altman DG. Measuring inconsistency in meta-analyses. BMJ (Clinical research ed). 2003;327(7414):557-60.

17. Danilovic DLS, Castroneves LA, Suemoto CK, Elias LO, Soares IC, Camargo RYA, Correa F, Hoff AO, Marui S. Is there a difference between minimal and gross extension into the strap muscles for the risk of recurrence in papillary thyroid carcinomas? Thyroid. 2020. https://doi.org/10.1089/thy.2019.0753.

18. Li G, Li R, Song L, Chen W, Jiang K, Tang H, Wei T, Li Z, Gong R, Lei J, et al. Implications of Extrathyroidal Extension Invading Only the Strap Muscles in Papillary Thyroid Carcinomas. Thyroid. 2020;30(1):57-64.

19. Park SY, Kim HI, Kim JH, Kim JS, Oh YL, Kim SW, Chung JH, Jang HW, Kim TH. Prognostic significance of gross extrathyroidal extension invading only strap muscles in differentiated thyroid carcinoma. The British journal of surgery. 2018;105(9):1155-62.

20. Song E, Kim WW, Jeon MJ, Sung TY, Song DE, Kim TY, Chung KW, Kim WB, Shong YK, Hong SJ, et al. Clinical Significance of Gross Invasion of Strap Muscles in Patients With 1- to 4-cm-Sized Papillary Thyroid Carcinoma Undergoing Lobectomy. Ann Surg Oncol. 2019;26(13):4466-71.

21. Radowsky JS, Howard RS, Burch HB, Stojadinovic A. Impact of Degree of Extrathyroidal Extension of Disease on Papillary Thyroid Cancer Outcome. Thyroid. 2014;24(2):241-4.

22. Arora N, Turbendian HK, Scognamiglio T, Wagner PL, Goldsmith SJ, Zarnegar R, Fahey TJ. Extrathyroidal extension is not all equal: Implications of macroscopic versus microscopic extent in papillary thyroid carcinoma. Surgery. 2008;144(6):942-7.

23. Jung SP, Kim M, Choe JH, Kim JS, Nam SJ, Kim JH. Clinical Implication of Cancer Adhesion in Papillary Thyroid Carcinoma: Clinicopathologic Characteristics and Prognosis Analyzed with Degree of Extrathyroidal Extension. World journal of surgery. 2013;37(7):1606-13.

24. Kim HI, Kim K, Park SY, Choe JH, Kim JH, Kim JS, Oh YL, Hahn SY, Shin JH, Ahn HS, et al. Refining the eighth edition AJCC TNM classification and prognostic groups for papillary thyroid cancer with lateral nodal metastasis. Oral Oncol. 2018;78:80-6.

25. Lamartina L, Grani G, Arvat E, Nervo A, Zatelli MC, Rossi R, Puxeddu E, 
Morelli S, Torlontano M, Massa M, et al. 8th edition of the AJCC/TNM staging system of thyroid cancer: what to expect (ITCO\#2). Endocr-Relat Cancer. 2018;25(3):L7-L11.

26. Clark $\mathrm{OH}$. Predictors of thyroid tumor aggressiveness. The Western journal of medicine. 1996;165(3):131-8.

27. Zhang X, Zhang L, Xue S, Wang P, Chen G. Predictive factors of lateral lymph node metastasis in solitary papillary thyroid microcarcinoma without gross extrathyroidal extension. Asian journal of surgery. 2019;42(4):563-70. 